\title{
Acetyl-DL-leucine improves gait variability in patients with cerebellar ataxia-a case series
}

\author{
Roman Schniepp ${ }^{1,2}$, Michael Strupp ${ }^{1,2}$, Max Wuehr ${ }^{2}$, Klaus Jahn ${ }^{2,3}$, Marianne Dieterich ${ }^{1,2}$, Thomas Brandt ${ }^{2,4}$ \\ and Katharina Feil ${ }^{1,2^{*}}$
}

\begin{abstract}
Acetyl-DL-leucine is a modified amino acid that was observed to improve ataxic symptoms in patients with sporadic and hereditary forms of ataxia. Here, we investigated the effect of the treatment with Acetyl-DL-leucine on the walking stability of patients with cerebellar ataxia (10x SAOA, 2x MSA-C, 2x ADA, 1x CACNA-1A mutation, 2x SCA 2, 1x SCA 1). Treatment with Acetyl-DL-leucine (500 mg; 3-3-4) significantly improved the coefficient of variation of stride time in 14 out of 18 patients. Moreover, subjective ambulatory scores (FES-I and ABC) and the SARA scores were also improved under treatment. Further prospective studies are necessary to support these class III observational findings.
\end{abstract}

Keywords: Gait variability, Acetyl-DL-leucine, Cerebellar ataxia, Speed dependency, symptomatic treatment

\section{Introduction}

Mobility impairments in patients with cerebellar ataxia (CA) are common. They are associated with a higher risk of falls, which often result in an increased fear of falling as well as in serious injuries [1]. Thus, symptomatic treatment options for patients with CA should aim to reduce the occurrence of falls by improving their dynamic balance. Gait variability measures which are linked to dynamic walking stability were found to be associated to falls in CA [2], thereby providing a novel and also quantitative measure for the assessment of ataxic gait disorders.

Acetyl-DL-leucine, a modified amino acid, was observed to improve ataxic symptoms in patients with sporadic and hereditary forms of ataxia [3], as well as in patients with Niemann-Pick Type C [4]. This effect was not found in a similar study performed in patients with sporadic, degenerative ataxia only [5].

Here we report on the effect of acetyl-DL-leucine on temporal gait variability in 18 patients with cerebellar ataxia (12 sporadic, 6 hereditary ataxia).

\footnotetext{
* Correspondence: katharina.feil@med.uni-muenchen.de

${ }^{1}$ Department of Neurology, University of Munich, Campus Großhadern, Marchioninistrasse 15, Munich 81377, Germany

${ }^{2}$ German Center for Vertigo and Balance Disorders (DSGZ), University of

Munich, Campus Großhadern, Marchioninistrasse 15, Munich 81377, Germany

Full list of author information is available at the end of the article
}

\section{Methods}

Patients were treated with acetyl-DL-leucine $5 \mathrm{~g} / \mathrm{d}$ without titration (500 mg tablets of Tanganil ${ }^{\mathrm{m}}$ ) for at least 4 weeks. Indication for the treatment with acetyl-DLleucine were the presence of cerebellar symptoms and abnormal findings in the domain "gait" of the Scale and Assessment of Ataxia (SARA). Exclusion criteria were concomitant diseases that affect locomotion. Twelve out of the 18 enrolled subjects received physiotherapeutic treatments with a weekly intensity of 20-60 min already before the pharmacological off-label treatment and also during it. Physiotherapy was not started new during the off-label treatment with acetyl-DL-leucine. All patients gave their informed consent for the compassionate offlabel use of acetyl-DL-leucine. The series was conducted by individual treatment efforts of patients after they had given informed consent. Eight of these patients had been part of our previous case series [3]. Treatment of patients took place from 2013 to 2014 at the Department of Neurology and at the German Center for Vertigo and Balance Disorders. We performed gait analysis using a pressure-sensitive carpet (GAITRite ${ }^{\circ}$ CIR System, Havertown, USA; sensor area $6.70 \mathrm{~m}$, sampling rate $120 \mathrm{~Hz}$ ). The walking pattern of all patients was recorded at three different walking speeds (slow, preferred, and maximal fast). Each speed was tested four times. 
Gait velocity and temporal gait variability quantified by the coefficient of variation $(\mathrm{CV})$ of stride time were analyzed. The tests were performed before starting of treatment and during treatment at time point 1 (7-14 days) and time point 2 (28-42 day). A repeated measure ANOVA (rmANOVA) with the covariate "walking speed" and the inner-subject factor "time point" was performed. A Sheffé posthoc analysis was performed.

\section{Results}

Clinical examination of patients revealed a moderate severity of symptoms (Scale for Assessment and Rating of Ataxia (SARA) [6]) and significant gait impairments (Functional Gait Assessment (FGA) [7] (Table 1).

Compared to the baseline measurement, walking speed was not significantly different under treatment at 1-2 weeks or at $>4$ weeks of treatment. For CV of stride time, we found a significant reduction in the rmANOVA model ( $\mathrm{df}=2, F=6.64, p<.01)$, in the posthoc analysis only for the condition of slow walking $(F=.3 .41, p<.05)$. Here, 14 patients showed a reduction of CV of stride time by $36 \%$ during 1-2 weeks of treatment (1-2 weeks) and $49 \%$ during $>4$ weeks of treatment (Fig. 1).

Subjective ambulatory scores (FES-I and ABC) were also improved in 12 patients, all of which showed a reduction of temporal gait variability. The SARA score

Table 1 Clinical characteristics and ambulatory functions of the patients

\begin{tabular}{ll}
\hline Demographic characteristics & 18 (9 women) \\
$\mathrm{N}$ & $58.83 \pm 12.0$ \\
mean age (years) & $1.73 \pm 0.07$ \\
height (m) & $72.6 \pm 6.1$ \\
weight (kg) & $72.0 \pm 29.3$ \\
duration of symptoms (months) & $12[8 ; 20]$ \\
median SARA score [range] & \\
Etiology of ataxia & $10 \times \mathrm{SAOA}, 2 \times \mathrm{MSA}-\mathrm{C}$ \\
sporadic & $2 \times \mathrm{ADA}, 1 \times \mathrm{CACNA}-1 \mathrm{~A}$ mutation, \\
hereditary & $2 \times \mathrm{SCA} 2,1 \times \mathrm{SCA} 1$ \\
& \\
Walking performance & $19[6 ; 30]$ \\
median FGA score [range] & 12 \\
Ambulatory status & 4 \\
independent & 2 \\
Intermediate use of aides & \\
prevalent use of aides &
\end{tabular}

Legend: Abbreviations: FGA Functional Gait Assessment [7], SARA Scale for the Assessment and Rating of Ataxia [6], SAOA Sporadic Adult Onset Cerebellar Atrophy, ADA Autosomal Dominant Ataxia, CACNA-1A Calcium Channel. voltage-dependent Alpha 1A subunit (P/Q type), SCA Spino Cerebellar Ataxia MSA-C multiple system atrophy with cerebellar features

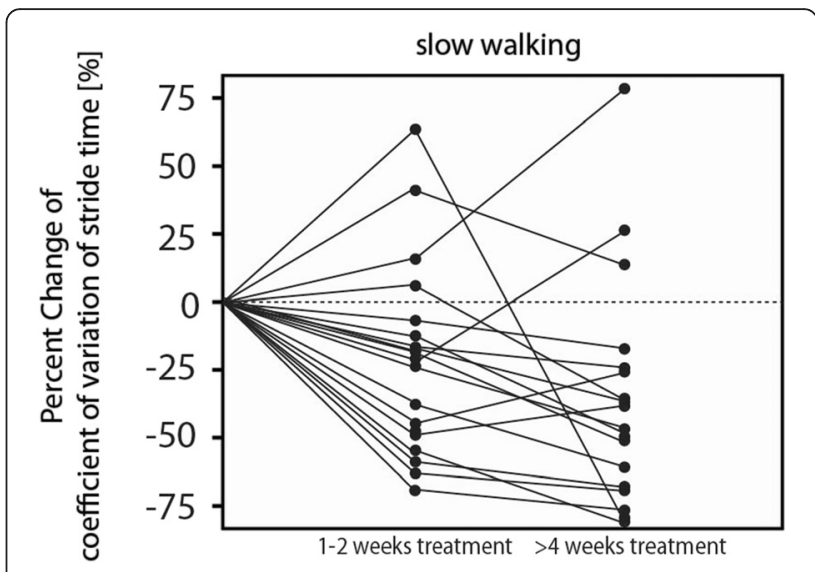

Fig. 1 Percent change of the coefficient of variation (CV) of stride time under continuous treatment with Acetyl-DL-leucine. Legend: Scatter plot with individual courses of temporal gait variability before and under treatment with Acetyl-DL-leucine.14 patients showed a reduction of CV of stride time by $36 \%$ during $1-2$ weeks of treatment ( $1-2$ weeks) and $49 \%$ during $>4$ weeks of treatment

showed an improvement of the median from 13 to 11 points $(F=3.25, p<.05)$ (for further information see Table 2). This effect did not correlate with the change of the $\mathrm{CV}$ of stride time.

\section{Discussion}

In summary, Acetyl-DL-leucine reduced temporal gait variability in 14 out of 18 patients with sporadic or hereditary CA. Acetyl-DL-leucine may thus offer a new and complementary symptomatic treatment option for cerebellar gait disorders.

The improvement of variability was restricted to the condition of slow walking, where walking stability is thought to critically rely on the sensory integration function of the cerebellum $[8,9]$. In this context it is important to note that instabilities at slow walking are highly associated with a history of falls in patients with CA [2]. In animal models, Acetyl-DL-leucine has been shown to interact with structures of central vestibular function $[10,11]$. An FDG-PET-study in humans demonstrated increased glucose metabolism in responders to AcetylDL-leucine treatment in central sensorimotor integration centers [12]. Thereby it may indirectly influence sensory processing of the cerebellum for maintaining dynamic stability during walking. We did not find a correlation of gait variability improvement with the improvement of clinical scores (FGA, SARA), although the SARA score did show a significant improvement under treatment. Taking together the present findings and a recent study on the improvement of gait variability under treatment with 4-aminopyridine [13, 14], support the idea that specific functions of walking might be 
Table 2 Patient characteristics, categorized by patient number, gender, age, etiology, duration of disease, neuro-ophthalmological findings, participation in a previous case study [3] as well a clinical outcome scores before and under treatment with Acetyl-DL-leucine

\begin{tabular}{|c|c|c|c|c|c|c|c|}
\hline ID & $\begin{array}{l}\text { Age } \\
\text { gender }\end{array}$ & $\begin{array}{l}\text { ataxia } \\
\text { type }\end{array}$ & duration & neuro-ophthalmological findings & $\begin{array}{l}\text { SARA } \\
\text { pre }\end{array}$ & $\begin{array}{l}\text { SARA } \\
\text { post }\end{array}$ & $\begin{array}{l}\text { Study } \\
\text { Strupp et al. } 2013\end{array}$ \\
\hline 1 & $73, m$ & SAOA & $>24$ & 8 & 12.5 & 9.5 & \\
\hline 2 & $57, w$ & $\mathrm{ADA}$ & $>120$ & 1,3 & 8.0 & 6.0 & $x$ \\
\hline 3 & $73, w$ & SAOA & 24 & $1,2,4$ & 20.0 & 15.0 & \\
\hline 4 & $54, w$ & MSA-C & 36 & $1,2,5,8$ & 13.0 & 12.0 & \\
\hline 5 & $59, \mathrm{~m}$ & SCA 2 & 56 & $1,2,7$, slow saccades & 16.0 & 12.5 & \\
\hline 6 & $23, \mathrm{~m}$ & SCA 2 & 36 & $1,2,7$, slow saccades & 13.0 & 9.0 & $x$ \\
\hline 7 & $68, w$ & $\mathrm{ADA}$ & $>120$ & $1,2,3,6,7,8$, hypometric saccades & 17.0 & 11,0 & $x$ \\
\hline 8 & $60, m$ & SAOA & 24 & 1,6 & 14.0 & 12.5 & $x$ \\
\hline 9 & $68, w$ & MSA-C & 36 & 1,2 & 10,0 & 9.5 & \\
\hline 10 & $54, \mathrm{~m}$ & SAOA & 60 & $1,2,6,8$ & 12.0 & 9.5 & \\
\hline 11 & $63, w$ & SAOA & $>120$ & $1,2,3,4,6,7$ & 14.5 & 12.5 & $x$ \\
\hline 12 & $51, \mathrm{~m}$ & SAOA & 36 & $1,2,3,6,7$ hypometric saccades & 14.0 & 12.0 & $x$ \\
\hline 13 & $51, w$ & EA 2 & 24 & 1 & 17.0 & 16.0 & \\
\hline 14 & $49, \mathrm{~m}$ & SAOA & 36 & $1,2,6,7$ & 16.5 & 15.5 & \\
\hline 15 & $63, w$ & SAOA & $>120$ & $1,2,3,4,6,7$ & 11.5 & 7.0 & $x$ \\
\hline 16 & $67, \mathrm{~m}$ & SAOA & 48 & $1,2,4$ & 13.5 & 11.5 & \\
\hline 17 & $60, m$ & SAOA & 36 & $1,3,5,8$ & 16.0 & 12.5 & \\
\hline 18 & $47, w$ & SCA 1 & 36 & 1, 2, 5 (bilateral), 6, 7, hypermetric saccades & 17.0 & 9.5 & $x$ \\
\hline
\end{tabular}

Legend: Abbrevations: $\mathrm{m}$ man, w woman, SCA spino cerebellar ataxia, EA 2 episodic ataxia type 2, MSA-C multiple system atrophy with cerebellar features, SAOA sporadic adult-onset ataxia of unknown etiology, ADA autosomal dominant cerebellar ataxia, 1 saccadic smooth pursuit, 2 gaze-evoked nystagmus, 3 headshaking nystagmus, 4 rebound nystagmus, 5 pathological head-thrust test (uni- or bilateral), 6 impaired visual fixation suppression of the VOR, 7 pathological optokinetic reflex, 8 downbeat nystagmus

influenced by pharmacotherapy. These findings promote future randomized placebo-controlled, double-blinded clinical trials on the symptomatic treatment of $\mathrm{CA}$, such as the FACEG (Fampridine) and the ALCAT trial (Acetyl-DL-leucine).

Analysis of speed-dependent effects on gait variability showed that non-preferred slow (acetyl-DL-leucine) and fast (4-aminopyridine) walking speeds are most responsive to pharmacological treatment in patients with CA. Whereas aminopyridines have been shown to improve gait variability mainly during fast walking [13, 14], Acetyl-DL-leucine was effective in reducing gait variability during slow walking. These findings are in accordance to an earlier study of our group showing the amount of gait variability being highest during slow and fast walking modes [8]. Thus, future randomized, controlled trials should take the speed-dependency of gait variability in patients with CA in account. Moreover, it appears to be questionable whether the clinical assessment of gait performance as determined in the SARA is sufficient and sensitive enough to assess improvements of dynamic stability in patients with CA during therapeutic interventions, since slow walking modes are commonly not examined.
If considering the assessment of dynamic stability during walking, study protocols should also include prospective recording of falls and the examination of gait variability markers.

\section{Consent}

Written informed conset was obtained from the patients for publication of the manuscript, accompanying images and figures.

\section{Competing interest}

Michael Strupp, MD, FANA, FEAN, is Joint Chief Editor of the Journal of Neurology, Editor in Chief of Frontiers of Neuro-otology and Section Editor of F1000. He has received speaker's honoraria from Abbott, Actelion, Biogen, Eisai, GSK, Hennig Pharma, Pierre-Fabre, MSD, TEVA, and UCB. Michael Strupp worked as consultant for Abbott. The other authors report that they have no conflicts of interest.

\section{Authors' contributions}

RS research project organisation, conception of project, statistical analysis (design, execution), manuscript preperation (writing of the first draw, review), including medical writing for content. MS manuscript preparation, review and critique, including medical writing for content. MW statistical analysis, review of data. KJ manuscript preparation including review of manuscript. MD manuscript preparation, review and critique. TB manuscript reparation (review). KF execution, clinical evaluation of patients, recruitment of patients, conception of project. Manuscript preparation including medical writing for content. All authors read and approved the final manuscript. 


\section{Acknowledgment}

The Authors thank Judy Benson for copy editing the article. The work was supported by the Federal Ministry for Education and Science (BMBF, Nr. 80121000-49) and the German Hertie Foundation.

\section{Author details}

'Department of Neurology, University of Munich, Campus Großhadern, Marchioninistrasse 15, Munich 81377, Germany. ${ }^{2}$ German Center for Vertigo and Balance Disorders (DSGZ), University of Munich, Campus Großhadern, Marchioninistrasse 15, Munich 81377, Germany. ${ }^{3}$ Schoen Klinik Bad Aibling, Neurologie, Kolbermoorer Strasse 72, Bad Aibling 83043, Germany. ${ }^{4}$ Institute of Clinical Neurosciences, University of Munich, Campus Großhadern,

Marchioninistrasse 15, Munich 81377, Germany.

Received: 26 October 2015 Accepted: 22 March 2016

Published online: 12 April 2016

\section{References}

1. Fonteyn EM, et al. Prospective analysis of falls in dominant ataxias. Eur Neurol. 2013;69(1):53-7.

2. Schniepp R, et al. Increased gait variability is associated with the history of falls in patients with cerebellar ataxia. J Neurol. 2014;261(1):213-23.

3. Strupp $M$, et al. Effects of acetyl-DL-leucine in patients with cerebellar ataxia: a case series. J Neurol. 2013;260(10):2556-61.

4. Bremova T, et al. Acetyl-dl-leucine in Niemann-Pick type C: A case series. Neurology. 2015;85(16):1368-75.

5. Pelz JO, et al. Failure to confirm benefit of acetyl-DL-leucine in degenerative cerebellar ataxia: a case series. J Neurol. 2015;262(5):1373-5.

6. Schmitz-Hubsch, $T$ et al. Scale for the assessment and rating of ataxia: development of a new clinical scale. Neurology. 2006;66(11):1717-20.

7. Wrisley DM, Kumar NA. Functional gait assessment: concurrent, discriminative, and predictive validity in community-dwelling older adults. Phys Ther. 2010;90(5):761-73.

8. Schniepp R, et al. Locomotion speed determines gait variability in cerebellar ataxia and vestibular failure. Mov Disord. 2012;27(1):125-31.

9. Schniepp R, et al. The gait disorder in downbeat nystagmus syndrome. PLoS One. 2014;9(8):e105463.

10. Vibert N, Vidal PP. In vitro effects of acetyl-DL-leucine (tanganil) on central vestibular neurons and vestibulo-ocular networks of the guinea-pig. Eur J Neurosci. 2001;13(4):735-48.

11. Ferber-Viart C, Dubreuil C, Vidal PP. Effects of acetyl-DL-leucine in vestibular patients: a clinical study following neurotomy and labyrinthectomy. Audiol Neurootol. 2009;14(1):17-25.

12. Becker-Bense S, Feuerecker R, Xiong G, Feil K, Bartenstein P, Strupp M, Dieterich M. Effects of acetyl-DL-leucine on the cerebral activation pattern in cerebellar ataxia (FDG-PET study) - Oral Sessions No. 01201. Eur J Neurol. 2015;22:21-117.

13. Schniepp $R$, et al. 4-Aminopyridine improves gait variability in cerebellar ataxia due to CACNA 1A mutation. J Neurol. 2011;258(9):1708-11.

14. Schniepp R, et al. 4-aminopyridine and cerebellar gait: a retrospective case series. J Neurol. 2012;259(11):2491-3.

\section{Submit your next manuscript to BioMed Central and we will help you at every step:}

- We accept pre-submission inquiries

- Our selector tool helps you to find the most relevant journal

- We provide round the clock customer support

- Convenient online submission

- Thorough peer review

- Inclusion in PubMed and all major indexing services

- Maximum visibility for your research

Submit your manuscript at www.biomedcentral.com/submit
Biomed Central 